\title{
Erratum to: Evaluation of a new immunochromatographic test for Helicobacter pylori IgG antibodies in elderly symptomatic patients
}

\author{
Haim Shirin - Rafael Bruck - Gabriel Kenet - Zipora Krepel • \\ Joram Wardi · Simon Reif • Liliana Zaidel • Dikla Geva • \\ Yona Avni · Zamir Halpern
}

Published online: 20 November 2012

(C) Springer Japan 2012

Erratum to: J Gastroenterol (1999) 34:7-10

DOI 10.1007/s005350050209

The correct name of the fifth author should be given as Joram Wardi, not Yoram Wardi.

The online version of the original article can be found under doi:10.1007/s005350050209.

H. Shirin · R. Bruck $(\bowtie) \cdot$ G. Kenet · Z. Krepel · J. Wardi .

S. Reif · Y. Avni · Z. Halpern

Department of Gastroenterology,

The E. Wolfson Medical Center,

Holon, and the Sackler Faculty of Medicine,

Tel-Aviv University, Tel-Aviv, Israel

e-mail: rafib@tasmc.health.gov.il

L. Zaidel

Department of Pathology, The E. Wolfson Medical Center,

Tel-Aviv, Israel

D. Geva

Department of Biostatistics, The E. Wolfson Medical Center,

Tel-Aviv, Israel 